\title{
Phase coherence in a random one-dimensional system of interacting fermions: A density-matrix renormalization-group study
}

\author{
Peter Schmitteckert and Ulrich Eckern \\ Institut für Physik, Universität Augsburg, D-86135 Augsburg, Germany
}

(Received 27 February 1996)

\begin{abstract}
Using the density-matrix renormalization-group algorithm, we study the model of spinless fermions with nearest-neighbor interaction on a ring in the presence of disorder. We determine the spatial decay of the density induced by a defect (Friedel oscillations), and the phase sensitivity of the ground-state energy $\Delta E=(-)^{N}[E(\phi=0)-E(\phi=\pi)]$, where $\phi=2 \pi \Phi / \Phi_{0}(N$ is the number of fermions, $\Phi$ the magnetic flux, and $\Phi_{0}=h / e$ the flux quantum), for a disordered system versus the system size $M$. The quantity $\ln (M \Delta E)$ is found to have a normal distribution to a good approximation. The "localization length" decreases (increases) for a repulsive (attractive) interaction. [S0163-1829(96)05624-X]
\end{abstract}

\section{INTRODUCTION}

About five decades after the first theoretical discussion of orbital magnetism of free electrons on a ring, ${ }^{1}$ this phenomenon - or " "persistent"' current — has been observed in mesoscopic metallic ${ }^{2,3}$ and semiconducting ${ }^{4,5}$ ring structures. At present, the magnitude of the effect - experimental results are much larger than theory predicts - is not well understood. It was suggested immediately after the experiment that the constraint of local charge neutrality, imposed by the electron-electron interaction, could lead to a considerable enhancement of the current, ${ }^{6,7}$ but these results, at least for single rings, were not conclusive, ${ }^{8}$ and were not confirmed numerically ${ }^{9}$ (see also, e.g., Refs. 10 and 11).

Considering electrons on a ring, the ground-state energy $E(\phi)$ depends on the boundary condition, characterized by the phase $\phi$ ( $\phi=0$ corresponds to periodic, and $\phi= \pm \pi$ to antiperiodic boundary conditions). Alternatively, the boundary condition can be interpreted as arising from a magnetic flux $\Phi$ provided we identify $\phi$ with $2 \pi \Phi / \Phi_{0}$, where $\Phi_{0}=h / e$ denotes the flux quantum. Clearly, $E(\phi)$ is periodic with period $2 \pi$. The energy difference between periodic and antiperiodic boundary conditions, $\Delta E$, the persistent current, $I \sim-E^{\prime}(\phi)$, and the charge stiffness (the "Drude weight" $), D \sim E^{\prime \prime}(\phi=0)$, are a measure of the phase sensitivity of the system (a comprehensive discussion is given in Ref. 12). In view of the discrepancy between experiment and theory, in particular for the metallic samples, ${ }^{2,3}$ it is important to understand further the interplay between interaction and disorder. Progress has been made for simple one-dimensional models $;^{12}$ we concentrate on these in the following.

We investigate the standard model of spinless fermions on a ring, with nearest-neighbor interaction $V$ (in units of the hopping amplitude), the lattice constant is unity, $M$ denotes the number of sites (i.e., the system size), and $N$ the fermion number. We restrict ourselves to the case of half filling, $N=M / 2$, and consider on-site disorder. The model is described by the Hamiltonian $\mathcal{H}=\mathcal{H}_{K}+\mathcal{H}_{V}+\mathcal{H}_{I}$, with

$$
\mathcal{H}_{K}=-\sum_{j}\left(\mathrm{e}^{l \varphi} c_{j+1}^{\dagger} c_{j}+\text { H.c. }\right)
$$

and

$$
\mathcal{H}_{V}=V \sum_{j} n_{j} n_{j+1}
$$

contrast to the "generic" defects described by the random on-site energies $\left\{\epsilon_{j}\right\}$, it is also possible to construct integrable models ${ }^{13}$ with "transparent" impurities, which decrease the phase sensitivity even though there is no wavefunction localization. The distinction between "integrable" and "nonintegrable"' is also most important for the temperature dependence of the Drude weight, $D .{ }^{14}$ We remark that the clean case is well studied by exact methods (see, e.g., Refs. 15 and 16).

\section{THE DENSITY-MATRIX RENORMALIZATION-GROUP ALGORITHM}

The density-matrix renormalization-group (DMRG) algorithm, introduced by White, ${ }^{17}$ is a numerical technique that allows reliable results for one-dimensional quantum lattice models, of a size (up to a few hundred sites) much larger than accessible by exact diagonalization methods, to be obtained. The algorithm can, in short, be characterized as a "'projected diagonalization,', where the subspace to be projected onto is determined by the most probable eigenstates of a density matrix. Start, for example, with a reasonable representation of an $s$-site system, using $m$ relevant states. Then add one site, $s \rightarrow s+1$, and supplement the system by an "environment," namely, the sites $s+2, \ldots, 2(s+1)$. The basis of $(2 m)^{2}$ states formed in this way is used to determine the ground state of $\mathcal{H}_{2 s+2}$. (The factor "two" appears here since we have two states for each site.) The density matrix of the "system" determines the $m$ most important states, onto which all relevant operators are projected. Then add another site, $s+1 \rightarrow s+2$, and proceed. 


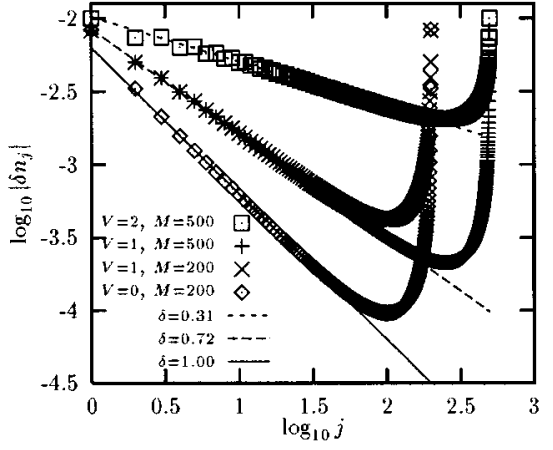

FIG. 1. Decay of the Friedel oscillations induced by weak impurities, located symmetrically at the ends of the chain $\left(\epsilon_{1}=-\epsilon_{M}=0.01\right)$. The increase for large $j$ arises due to the finiteness of the chain. The calculations are performed at half filling, $N=M / 2$, keeping $m=120$ (200) states per block for $M=200(500)$. The amplitude of the oscillation vanishes for $\epsilon_{1} \rightarrow 0$.

The result of this "infinite lattice" algorithm is the basis for the "finite lattice" algorithm, which is needed in order to treat nonreflection symmetric systems. (i) The infinite lattice algorithm is used to find a representation of all $s$-site systems with $s$ up to $M-3$. (ii) Then consider the sites $1, \ldots, s$ as a system, add two sites, and take as the environment the $s+3, \ldots, M$ system. (iii) Proceed as described above. This process can be characterized as "sweeping through the lattice." With $m$ up to several hundred, we have achieved sufficient accuracy, even for questions as subtle as the phase sensitivity. The method works best for open boundary conditions, though there is no general problem (except enhanced computing time) to include twisted boundary conditions. Since the DMRG is a local method, disorder is easily included, though disorder averages of course require considerable computing time. For example, the data given in Fig. 3 are based on roughly $300 \mathrm{CPU}$ days on a high-end workstation. Further details, e.g., the adaption to nonreflection symmetric models, are discussed in Ref. 18.

\section{FRIEDEL OSCILLATIONS}

The decay of the density oscillations induced by a defect is a long-standing problem in solid state physics. This phenomenon, called Friedel or Ruderman-Kittel oscillations (depending on the context), is closely related to the singularity in the response function for wave vectors close to $2 k_{F}$. It is expected that asymptotically, the induced density decays as

$$
\delta n(x) \sim \frac{\cos \left(2 k_{F} x+\eta_{F}\right)}{x^{\delta}} .
$$

Using the DMRG, we have computed $\delta n(x)$ for a system of 200 sites and various interaction strengths and, as a test for the accuracy of our calculation, for systems with $M=500$ and $V= \pm 1,2$. The impurity is chosen antisymmetrically for technical reasons, $\epsilon_{1}=-\epsilon_{M}$. We consider a half-filled band, i.e., $2 k_{F} x_{j}=\pi j$, where $j$ is the distance (in units of the lattice spacing) from the defect. A sample of our results is

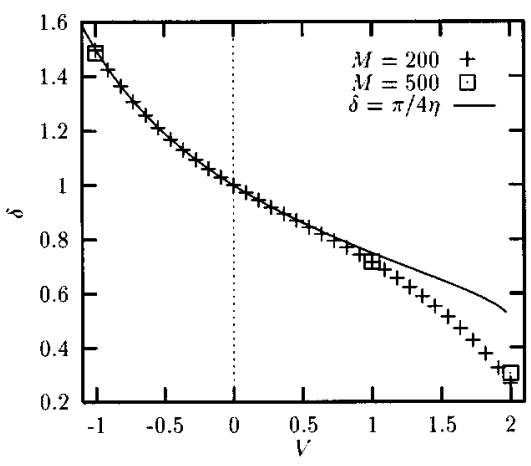

FIG. 2. The exponent $\delta$ vs interaction, for the same impurity as in Fig. 1. The continuous line is the asymptotic result (Ref. 20).

shown in Fig. 1, where we plot, on a logarithmic scale, the magnitude $\left|\delta n_{j}\right|$ versus distance. Clearly, it is possible to extract the exponent $\delta$ without difficulty. We emphasize that the algebraic decay starts already at a few lattice site sites. Typically, we have used a basis of $m=120$ (200) states for the $M=200$ (500) system, and performed four sweeps through the lattice.

The exponent $\delta$ as a function of the nearest-neighbor interaction $V$ is given in Fig. 2. The exponent $\delta$ deceases with increasing repulsive interaction, and increases with attractive interaction, compared to the value for noninteracting fermions, $\delta=1$ (one dimension).

Qualitatively, this trend agrees with the prediction ${ }^{19}$ based on the Luttinger liquid. In a recent work, ${ }^{20} \delta$ was related to the "dressed charge" of the (clean) model, with the result $\delta=Z^{2}=\pi / 4 \eta$, where $\eta$, related to $V$ through $V=-2 \cos (2 \eta)$, parametrizes the interaction. The expression $\delta=\pi / 4 \eta$ is also shown in Fig. 2, and is in almost perfect agreement with our numerical data, except for $V>1$ where we find that the oscillations decay more weakly than predicted. This seems to be related to the crossover (for a weak impurity, and $V>0$ ) found in Ref. 19, i.e., for the system sizes studied we may not yet be in the asymptotic regime. We have preliminary results showing that for a strong impurity, $\delta$ tends to increase towards the asymptotic result given in Ref. 20.

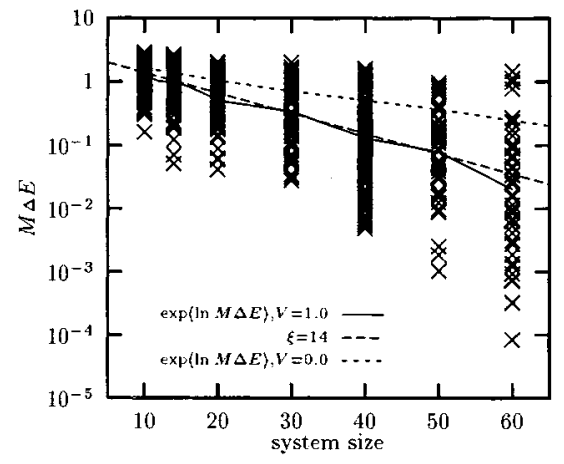

FIG. 3. Phase sensitivity of the ground-state energy vs system size for a repulsive interaction $(V=1.0, W=2.0, N=M / 2)$. This value of $W$ corresponds to $\xi_{0} \approx 26$ for the noninteracting case. The decay length is $\xi \approx 14$. 


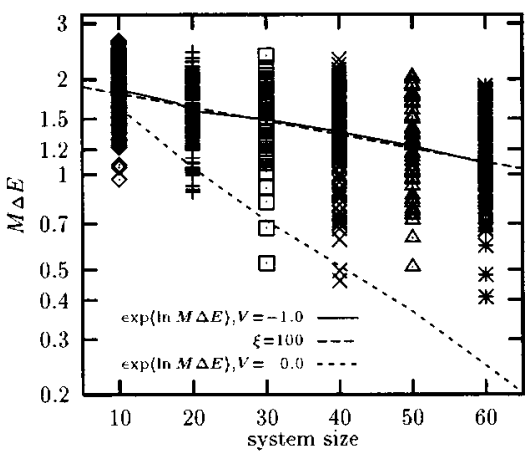

FIG. 4. Phase sensitivity vs system size for an attractive interaction $(V=-1.0, W=2.0, N=M / 2)$. The decay length is $\xi \approx 100$.

\section{PHASE SENSITIVITY}

Concerning the phase sensitivity of the ground-state energy, let us recall the free-electron result for odd $N$,

$$
[E(\phi)-E(0)]_{0}=\frac{\hbar v_{F}}{2 \pi L} \phi^{2}
$$

to be continued periodically outside the interval $-\pi \cdots \pi$. For an even number of particles, $\phi \rightarrow \phi-\pi$ in this equation. In our units, and for a half-filled band, we have $\hbar v_{F} / L \rightarrow 2 / M$; thus $\Delta E_{0}=(-1)^{N}[E(0)-E(\pi)]_{V=0}=\pi / M$ for the clean, noninteracting system.

Disorder is introduced by taking the on-site energies $\left\{\epsilon_{j}\right\}$ as random quantities, uniformly distributed over the range $-W / 2 \cdots W / 2$, which corresponds for free fermions to a conductance localization length $\xi_{0} \approx 105 / W^{2}$, i.e., the average conductance decreases as $\langle g\rangle \sim \exp \left(-2 M / \xi_{0}\right)$ for a wire of length $M .^{21}$ We considered $W=2$ only, hence $\xi_{0} \approx 26$. Naturally, we first studied noninteracting fermions, using 500 samples, i.e., 500 different realizations of the disorder for each system size. Interestingly, we find $\left\langle\Delta E_{0}\right\rangle \sim\langle g\rangle^{1 / 4}$, but we also see that the $\Delta E$ distribution is rather asymmetric, in contrast to the distribution of $\ln (M \Delta E)$. The average $\left\langle\ln \left(M \Delta E_{0}\right)\right\rangle$ decreases as const $-M / \xi$, with $\xi \approx 29$, close to the conductance localization length.

In Fig. 3 we present our results, i.e., $M \Delta E$ versus $M$, for a repulsive interaction, $V=1.0$. The $40-(50-, 60-)$ site systems have been calculated using $m=190(375,375)$ states and performing three finite lattice sweeps. The accuracy is better than $10^{-5}$ for the 60 -site systems. The phase sensitivity $\Delta E$ is positive for all samples. The dashed line, obtained by fitting $\langle\ln (M \Delta E)\rangle$ as described above, represents $-M / \xi$ with $\xi \approx 14$, about half of the free-fermion value. The error in $\xi$ may be about $20 \%$. (We feel that for $M=60$, the number of samples used, $\approx 50$, may not be sufficient.) The shortdashed line shows, for comparison, the decay of $\langle\ln (M \Delta E)\rangle$ for the noninteracting case.

In contrast, the phase sensitivity is strongly enhanced for an attractive interaction, as can be seen from Fig. 4 $(V=-1.0)$. The dashed line, again, is obtained by fitting $\langle\ln (M \Delta E)\rangle$ with $-M / \xi$, leading to $\xi \approx 100$. As an example, we have studied a 40 -site system in more detail, for a repul-

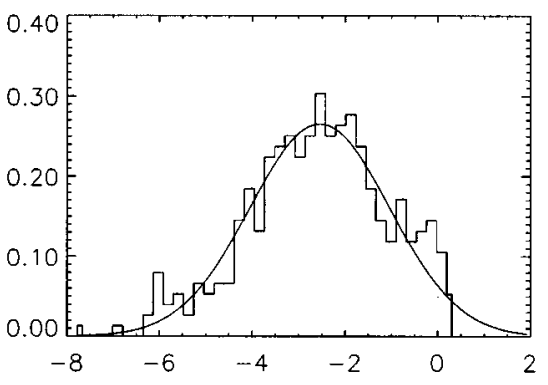

FIG. 5. Distribution of $\ln (M \Delta E)$ for $V=1.2$ on the basis of 353 samples. We find $\langle\ln M \Delta E\rangle=-2.54$ and $\sigma=1.5$ from our data. The continuous line is the corresponding Gauss curve.

sive interaction $(V=1.2)$. Using $\approx 350$ samples, we find a reasonably smooth distribution of $\ln (M \Delta E)$ values, as shown in Fig. 5. These data can be fitted well with the corresponding Gauss curve, computed from the average and the variance as given in the figure caption; these values were computed from their definition.

\section{CONCLUSIONS}

In conclusion, we have demonstrated that, using the DMRG algorithm, it is possible to obtain very accurate results for the ground-state properties of interacting onedimensional systems with defects, as, e.g., is apparent from the comparison of numerical and analytical results for the decay of the Friedel oscillations. In particular, system sizes of a few hundred sites are sufficient to obtain asymptotic results, except when the decay is very slow, i.e., $\sim x^{-1 / 2}$ or slower. Our results for the phase sensitivity are consistent with what is expected for spinless fermions (see Ref. 12 and reference therein). The attractive and the repulsive ground states both contain density fluctuations, but the attractive ground state also contains superconducting fluctuations. The latter screen the disorder, leading to an increase of the phase senstivity, i.e., a localization length larger than in the noninteracting case, for an attractive interaction. On the other hand, the phase senstivity is reduced for a repulsive interaction. As pointed out in Ref. 12, however, these trends are just opposite to what should be expected for "realistic" models, i.e., models that tend to homogenize the density for a repulsive interaction (as, e.g., is the case for the Hubbard model). We plan to study this question further. We are not aware, however, of any evidence that the phase sensitivity can become larger than in the clean, noninteracting case.

\section{ACKNOWLEDGMENTS}

We thank Karen Hallberg, Reinhold Egger, and Peter Schwab for stimulating discussions. The calculations were performed on an IBM SP2 at the Leibniz-Rechenzentrum Munich. We thank Christian Schaller and Matthias Brehm from the LRZ for the support concerning the SP2 cluster. Work supported by the Deutsche Forschungsgemeinschaft (Forschergruppe HO 955/2-1). 
${ }^{1}$ F. Hund, Ann. Phys. (Leipzig) 32, 102 (1938) [English translation, Ann. Phys. 5, 1 (1996)].

${ }^{2}$ L.P. Lévy, G. Dolan, J. Dunsmuir, and H. Bouchiat, Phys. Rev. Lett. 64, 2074 (1990).

${ }^{3}$ V. Chandrasekhar et al., Phys. Rev. Lett. 67, 3578 (1991).

${ }^{4}$ D. Mailly, C. Chapelier, and A. Benoit, Phys. Rev. Lett. 70, 2020 (1993).

${ }^{5}$ B. Reulet, M. Ramin, H. Bouchiat, and D. Mailly, Phys. Rev. Lett. 75, 124 (1995).

${ }^{6}$ V. Ambegaokar and U. Eckern, Phys. Rev. Lett. 65, 381 (1990).

${ }^{7}$ A. Schmid, Phys. Rev. Lett. 66, 80 (1991).

${ }^{8}$ U. Eckern and A. Schmid, Europhys. Lett. 18, 457 (1992); Ann. Phys. (Leipzig) 2, 180 (1992); see also R.A. Smith and V. Ambegaokar, Europhys. Lett. 20, 161 (1992).

${ }^{9}$ M. Abraham and R. Berkovits, Phys. Rev. Lett. 70, 1509 (1993).

${ }^{10}$ B.L. Altshuler, in Nanostructures in Mesoscopic Systems, edited by W.P. Kirk and M.A. Reed (Academic, San Diego, 1992), p. 405.

${ }^{11}$ P. Kopietz, Phys. Rev. Lett. 70, 3123 (1993); see also G. Vignale, ibid. 72, 433 (1994); A. Altland and Y. Gefen, ibid. 72, 2973 (1994).
${ }^{12}$ T. Giamarchi and B.S. Shastry, Phys. Rev. B 51, 10915 (1995).

${ }^{13}$ P. Schmitteckert, P. Schwab, and U. Eckern, Europhys. Lett. 30, 543 (1995); A. Punnoose, H.P. Eckle, and A. Römer (unpublished).

${ }^{14}$ H. Castella, X. Zotos, and P. Prelov̌sek, Phys. Rev. Lett. 74, 972 (1995).

${ }^{15}$ C.J. Hamer, G.R.W. Quispel, and M.T. Batchelor, J. Phys. A 20, 5677 (1987).

${ }^{16}$ B.S. Shastry and B. Sutherland, Phys. Rev. Lett. 65, 243 (1990); B. Sutherland and B.S. Shastry, ibid. 65, 1833 (1990).

${ }^{17}$ S.R. White, Phys. Rev. Lett. 69, 2863 (1992); S.R. White and R.M. Noack, ibid. 68, 3487 (1992); S.R. White, Phys. Rev. B 48, 10345 (1993).

${ }^{18}$ P. Schmitteckert, Ph.D. thesis, Universität Augsburg, http:// www.Physik.Uni-Augsburg.de/ peters (1996).

${ }^{19}$ R. Egger and H. Grabert, Phys. Rev. Lett. 75, 3505 (1995).

${ }^{20}$ Y. Wang, J. Voit, and Fu-Cho Pu (unpublished).

${ }^{21}$ M. Kappus and F.J. Wegner, Z. Phys. B 45, 15 (1981); G. Czycholl, B. Kramer, and A. MacKinnon, ibid. 43, 5 (1981). 